\title{
Influence of Piperine on Pioglitazone Metabolism and Pk/Pd: Diabetes Mellitus
}

\section{Prasad Neerati ${ }^{1 *}$ and Sudhakar A Yakkanti ${ }^{2}$}

${ }^{1}$ Department of Pharmacology, University College of Pharmaceutical Sciences, Kakatiya University, India

${ }^{2}$ Cell signaling Laboratory, Bioscience Division, Center for Cancer and Metabolism, SRI International, USA

\begin{abstract}
Background: Piperine is an alkaloidal compound and is an active constituent of black and long peppers. Piperine is known to inhibit cytochrome P- 450 isoforms. The objective was to determine the effects of dietary piperine on the pharmacokinetics and pharmacodynamics of pioglitazone.

Methods and Results: Set one non diabetic study, there are three groups, as 1,2 and 3 composed each of four Albino rabbits $(2-3 \mathrm{~kg})$. In the next set, another similar three groups named group 4 group 5 and group 6 were selected to investigate the influences in diabetic group after receiving alloxan monohydrate $(80 \mathrm{mg} / \mathrm{kg}$, i.v). Group 1 and group 2; group 4 and group 5 received pioglitazone (10 mg/kg; po) and piperine $(20 \mathrm{mg} / \mathrm{kg}$; po) respectively. Group 3 and group 6, we investigated herb drug interactions for single dose and multiple dose interactions on normal and diabetic rabbits with piperine for single day and for eight days. On the last day of piperine pre-treatment, pioglitazone was given. $1 \mathrm{ml}$ blood samples collected intravenously via marginal ear vein, at the pre set time points and PK \& PD parameters were measured. Serum levels of pioglitazone measured using RP-HPLC and glucose levels measured. Significant changes observed under multiple dose pre-treatment of piperine. $A U C_{\text {tot }}$ of pioglitazone significantly increased in normal and diabetic rabbits. The biological half-life $\left(\mathrm{t}_{12}\right)$ was increased whereas clearance was decreased. Maximum percentage glucose reduction was increased significantly in both single and multiple dose pre-treatment of piperine in diabetic rabbits. The observations suggest an interaction due to metabolic inhibition of CYP enzymes.
\end{abstract}

Conclusion: Piperine increases the bioavailability of pioglitazone in normal and diabetic rabbits. Piperine, pioglitazone combination has a beneficial effect in diabetes but it may require special care and should be given under medical supervision with appropriate dose combinations in severe diabetes.

Keywords: Piperine; Diabetes Mellitus; Alloxan; Pk/Pd; Pioglitazone; HPLC

\section{Introduction}

In some studies piperine is known to inhibit human CYP2C8, CYP3A4 and P-glycoprotein and the enzymes more important for the metabolism of pioglitazone and transport of xenobiotics [1-3]. Piperine can improve the bioavailability of many drugs and decrease the elimination of the drugs and finally improves the biological effectiveness [2]. Major population from America taking herbal medications without informing their medical doctors [4]. Piperine has been found to have anti diabetic activity per se [5]. However, as the metabolism of pioglitazone is inhibited by piperine there is the chance to improve the therapy with this combination.

Pioglitazone is metabolised by CYP3A4 [6] and its metabolism is inhibited by piperine. So there is the chance to potential interactions between piperine and pioglitazone combined usage. Pioglitazone can cause several complications during the treatment of the diabetes mellitus and the combination can reduce the dose and also the complications.

Investigators have predicted that India will have the greatest increase in diabetes and will have the largest number of diabetic patient in the world [7]. It has been reported that Asian Indians have an ethnic susceptibility to type 2 diabetes. The literature indicates that the prevalence of the metabolic syndrome and particularly diabetes is high among migrant Asian Indian and is rising very rapidly even within the Indian sub-continent. Recent WHO reports show that, India already has the largest number of the diabetic patient compared to any given company.

\section{Materials and Method}

\section{Animals and diet}

Albino white rabbits of male weighing 2-3 $\mathrm{kg}$ were used in the study. They were maintained under standard laboratory condition at ambient temperature. They were fed with pellet diet and water ad libitum. The animals were fasted for overnight before experiment and during the experiment they were withdrawn from food and water. The in vivo experimental protocol was approved (IAEC/11/UCPSc/ KU/2011) by the Institutional Animal Ethical committee Kakatiya University, Warangal.

\section{Drugs and chemicals}

Pioglitazone and rosiglitazone (internal standard) were the kind gift samples from Dr. Reddy's Lab (Hyderabad, India). Piperine supplied by Sinthite Pharma, (Kerala,India). Alloxan monohydrate from SigmaAldrich (Bangalore, India). Glucose estimation kits were supplied by Excel diagnostics pvt.ltd (Hyderabad, India). Orthophosphoric acid

*Corresponding author: Neerati Prasad, Assistant Professor of Pharmacy, DMPK \& Clinical Pharmacology Division, Department of Pharmacology, University College of Pharmaceutical Sciences, Kakatiya University, Warangal , AP, India Tel: 91-9494812120; Fax: 08702453508; E-mail: prasadneerati@gmail.com

Received February 24, 2014; Accepted March 29, 2014; Published April 04, 2014

Citation: Neerati P, Yakkanti AS (2014) Influence of Piperine on Pioglitazone Metabolism and Pk/Pd: Diabetes Mellitus. J Diabetes Metab 5: 356 doi:10.4172/2155-6156.1000356

Copyright: ( 92014 Neerati P, et al. This is an open-access article distributed under the terms of the Creative Commons Attribution License, which permits unrestricted use, distribution, and reproduction in any medium, provided the original author and source are credited. 
Citation: Neerati P, Yakkanti AS (2014) Influence of Piperine on Pioglitazone Metabolism and Pk/Pd: Diabetes Mellitus. J Diabetes Metab 5: 356 doi:10.4172/2155-6156.1000356

Page 2 of 7

analytical grade and HPLC grade acetonitrile, methanol and potassium dihydrogen phosphate supplied by Merck (Mumbai, India).

\section{HPLC analysis of pioglitasone}

Pioglitasone was estimated by an earlier reported modified reverse phase HPLC method [8,9]. HPLC system consisted of LC-10 ATVP solvent delivery module (Shimadzu, Kyoto, Japan), SPD-20AVP variable wavelength programmable UV/VIS spectrophotometric detector, a Class CR-10 Data processor and Reverse Phase C18 column (Wakosil II C-18, 250x4.6 mm, $5 \mu$ porous silica spheres) was used. Rheodyne injection port with a $20 \mathrm{ul}$ sample loop and Hamilton syringe $20 \mu \mathrm{L}$ was used.

\section{Chromatographic conditions}

Pioglitazone concentration was determined by slight modification of a method reported the mobile phase consists of $25 \mathrm{mM}$ Phosphate buffer (PH adjusted to 3 with orthophosphoric acid), acetonitrile \& methanol in a ratio of 55:37.5:7.5 $(\mathrm{v} / \mathrm{v} / \mathrm{v})$. The mobile phase was degassed \& filtered through $0.22 \mu \mathrm{m}$ membrane filter. The flow rate was $1.2 \mathrm{~mL} / \mathrm{min} \&$ the effluent was monitored at $269 \mathrm{~nm}$. The total run time of the method was set at $10 \mathrm{~min}$.

\section{Preparation of standard graph}

The stock solution of pioglitazone \& rosiglitazone were prepared in methanol at a concentration of $1 \mathrm{mg} / \mathrm{mL}$ each. Rosiglitazone was used as internal standard (IS). By appropriately diluting the stock solutions, different concentrations of pioglitazone $(0.01,0.05,0.1,0.5,1,5,10,50$ $\mu \mathrm{g} / \mathrm{mL})$ \& rosiglitazone $(10 \mu \mathrm{g} / \mathrm{mL})$ were prepared.

To a volume of $100 \mu \mathrm{L}$ of blank rabbit serum, $50 \mu \mathrm{L}$ of rosiglitazone (2.5 $\mu \mathrm{g}$ in methanol) solution as internal standard \& $100 \mu \mathrm{L}$ of acetonitrile were added to precipitate the proteins. The mixture was vortex mixed for $5 \mathrm{~min}$ after which it was centrifuged at $10,000 \times \mathrm{g}$ for $10 \mathrm{~min} .20 \mathrm{~L}$ of the supernatant was injected on to the HPLC system for analysis. The calibration curve was obtained by plotting peak area ratios of pioglitazone to rtosiglitazone ( $y$-axis) against pioglitazone concentration (x-axis).

\section{Preparation of test samples}

To a volume of $100 \mu \mathrm{L}$ of test rabbit serum, $50 \mu \mathrm{L}$ of rosiglitazone $(2.5$ $\mu \mathrm{g}$ in methanol) solution as internal standard \& $100 \mu \mathrm{L}$ of acetonitrile were added to precipitate the proteins. The mixture was vortex mixed for $5 \mathrm{~min}$ after which it was centrifuged at $10,000 \times \mathrm{g}$ for $10 \mathrm{~min}$. $20 \mathrm{~L} \mathrm{of}$ the supernatant was injected onto the HPLC system for analysis (Figure $1)$.

\section{Limit of detection and Limit of quantification}

Three calibration curves were obtained by spiking thrice, the standard dilutionss of pioglitazone in serum samples. 0.0782

The Equation of I calibration curve of pioglitazone: $y=0.179 x-$

The Equation of II calibration curve of pioglitazone: $y=0.179 x-$ 0.0793 0.0843

The Equation of III calibration curve of pioglitazone: $y=0.1778 x-$

$\sigma=0.003251$

$S=0.1786$

$\sigma \rightarrow$ Standard deviation of $y$ - intercepts of Calibration Curves.

$\mathrm{m} \rightarrow$ Mean of the slopes of Calibration Curves of Pioglitazone.

L.O.D $=3.3 \sigma / \mathrm{S}$

$=0.060072$

The L.O.D of Pioglitazone from the Equations of the three Calibration curves of Pioglitazone was found to be $0.060072 \mu \mathrm{g}$.

L.O.Q $=10 \sigma / \mathrm{S}$

$=0.18204$

$\sigma \rightarrow$ Standard deviation of y-intercepts of Calibration Curves.

$\mathrm{m} \rightarrow$ Mean of the slopes of Calibration Curves of Pioglitazone.

The L.O.Q of Pioglitazone from the Equations of the three Calibration curves of Pioglitazone was found to be $0.18204 \mu \mathrm{g}$.

Hence, the limit of detection and limit of quantification were both found to be within the range of the analytic levels in serum samples.

\section{Accuracy and precision}

Intra and inter-day precision expressed as percentage of standard

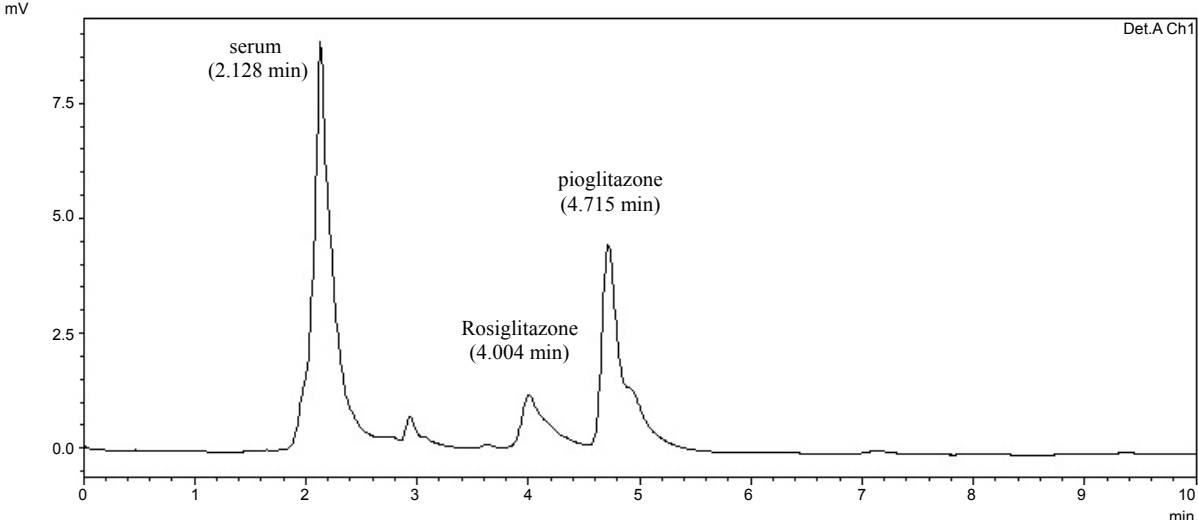

Serum (RT: $2.128 \mathrm{~min})$

Internal Standard: Rosiglitazone (RT: $4.004 \mathrm{~min}$ )

Drug: Pioglitazone (RT: $4.715 \mathrm{~min}$ )

Figure 1: HPLC chromatograms of pioglitazone in rabbit serum samples. 
deviation (\%RSD) and accuracy expressed as percentage of relative error (\%RE) obtained from three levels of quality control samples of Pioglitazone. The precision and accuracy of the method was established by using quality control samples at low, medium and high concentrations of $0.1,1 \& 10 \mu \mathrm{g} / \mathrm{mL}$ for Pioglitazone. All the samples were run in three replicates. Intra-day precision data was obtained by analyzing three sets of quality control samples in a single day, while the inter-day data was obtained by analyzing the quality control samples on three consecutive days of assay.

Assay precision was calculated using the formula:

$\% \mathrm{RSD}=(\mathrm{SD} / \mathrm{M}) \times 100$

$\mathrm{M} \rightarrow$ mean of the experimentally determined concentrations

$\mathrm{SD} \rightarrow$ standard deviation of $\mathrm{M}$.

Accuracy was calculated using the formula:

$\% \mathrm{RE}=[(\mathrm{E}-\mathrm{T}) / \mathrm{T}] 100$

$\mathrm{E} \rightarrow$ experimentally determined concentration.

$\mathrm{T} \rightarrow$ theoretical concentration.

Intra and inter day precision and accuracy of the determination of pioglitazone quality control samples $(n=4)$. The assay procedure was found to be precise and accurate.

\section{Calculations of pharmacokinetic parameters}

Non compartmental Pharmacokinetic analysis was carried out using Kinetica TM software (version 4.4.1 Thermo Electron Corporation, U.S.A). The following Pharmacokinetic parameters were calculated $\mathrm{C}_{\max }, \mathrm{T}_{\max }, \mathrm{t}_{1 / 2,}, \mathrm{AUC}_{\text {ton, }}, \mathrm{AUC}_{\text {tot }}$ and MRT.

\section{Statistical analysis}

Difference in between concentration time profiles; in between pharmacokinetic parameters; in between serum glucose levels and difference between over the entire range tested were analyzed by oneway ANOVA (Bonferoni post-test) (rockvilie1985). The differences were considered to be significant at $\mathrm{P}<0.05$.

\section{Induction of diabetes in rabbits}

Experimental diabetes in rabbits (2-3 kg) was induced by intravenous [10] injecting alloxan monohydrate $(80 \mathrm{mg} / \mathrm{kg})$ freshly disolve in normal saline in to marginal ear vein to the overnight fasted rabbits. $20 \%$ glucose solution was injected after 4-6 hrs. Rabbits were kept for the next $24 \mathrm{hrs}$ on $5 \%$ oral glucose solution, in their cages to prevent hypoglycemia. On the 5th day blood glucose level is increased the levels are measured by GOD POD method [11]. The levels of $230 \mathrm{mg} / \mathrm{dl}$ were considered as diabetic and control groups were given the same volume of normal saline.

\section{Study in normal rabbits}

Pharmacokinetic \& pharmacodynamic interaction study in normal rabbits

Following an overnight fast, rabbits were divided in to 3 groups each group contain 4 rabbits $(n=4)$.

Group I pioglitazone ( $10 \mathrm{mg} / \mathrm{kg}$; po.)

Group II piperine $(20 \mathrm{mg} / \mathrm{kg}$; po. ) followed by pioglitazone (10 $\mathrm{mg} / \mathrm{kg}$; po.)

(SDIs: Single dose interaction studies)
Group III piperine $\left(20 \mathrm{mg} / \mathrm{kg}\right.$; po.) for 7 days, on the $8^{\text {th }}$ day piperine $(20 \mathrm{mg} / \mathrm{kg}$; po.) and pioglitazone were administered. (MDIs: Multi Dose Interaction Studies)

Blood samples were collected from marginal ear vein [11] at time intervals at $0,0.5,1,2,4,8,24 \mathrm{hrs}$ in to eppendroff tubes the serum was separate by centrifugation using biofuge 13 (Heraeus instruments, germany) at $3000 \mathrm{~g} / 15 \mathrm{~min}$ separate serum and store at $-20^{\circ} \mathrm{C}$ until further analysis.

\section{Study in diabetic rabbits}

Pharmacokinetic \& pharmacodynamic interaction study in diabetic rabbits

Following an overnight fasted, rabbits were divided in to 3 groups each group contain 4 rabbits $(\mathrm{n}=4)$.

Group I pioglitazone ( $10 \mathrm{mg} / \mathrm{kg}$; po.)

Group II piperine (20 mg/kg; po.) followed by pioglitazone (10 $\mathrm{mg} / \mathrm{kg}$; po.)

(Single Dose Interaction Studies: SDIs; single time piperine exposure)

Group III Treating with piperine ( $20 \mathrm{mg} / \mathrm{kg}$; po.) for 7 days, on the $8^{\text {th }}$ day piperine $(20 \mathrm{mg} / \mathrm{kg}$; po.) and pioglitazone were administered. Piperine Exposure)

(Multiple Dose Interaction Studies : MDIs; Multiple Times

Blood samples were collected from marginal ear vein at time intervals at $0,0.5,1,2,4,8,24 \mathrm{hrs}$ in to eppendroff tubes the serum was separated by centrifugation using biofuge 13 (Heraeus instruments, germany) at $3000 \mathrm{~g} / 15 \mathrm{~min}$ separate serum and store at $-20^{\circ} \mathrm{C}$ until further analysis.

\section{Calculations of pharmacokinetic and pharmacodynamic parameters}

Non compartmental pharmacokinetic analysis was carried out using Kinetica TM software (version 4.4.1 Thermo Electron Corporation, U.S.A). The following Pharmacokinetic parameters were calculated: $\mathrm{C}_{\text {max }}, \mathrm{T}_{\text {max }}, \mathrm{AUC}_{\text {oton }}, \mathrm{AUC}_{\text {tot }}, \mathrm{AUMC}_{\text {oton, }}, \mathrm{AUMC}_{\text {to, }}, \mathrm{t}_{1 / 2}, \mathrm{MRT}, \mathrm{Cl}, \mathrm{Vd}$ and Vdss. Mean glucose levels and percentage reduction in blood glucose concentrations were determined for the pharmacodynamic data.

$$
\begin{aligned}
& \% \text { glucose reduction at } t \text { hour }=[(\mathrm{Gt}-\mathrm{G} 0) / \mathrm{Gt}] \times 100 \\
& \mathrm{Gt} \rightarrow \text { mean glucose levels at } \mathrm{t} \text { hour } \\
& \mathrm{G} 0 \rightarrow \text { mean glucose levels at } 0 \text { hour }
\end{aligned}
$$

\section{Statistical analysis}

The results expressed as mean $\pm \mathrm{SD}$. The difference in between concentration time profiles; in between pharmacokinetic parameters, in between serum glucose levels and difference between over the entire range tested were analyzed by one-way ANOVA (Bonferoni post-test). The differences were considered to be significant at $\mathrm{P}<0.05$.

\section{Results}

\section{Pharmacokinetic data}

Pharmacokinetics of pioglitazone in normal rabbits with piperine: The serum concentration levels of pioglitazone in normal rabbits were read out by substituting the peak area ratio values of each sample in the equation obtained from the calibration curve of 
Citation: Neerati P, Yakkanti AS (2014) Influence of Piperine on Pioglitazone Metabolism and Pk/Pd: Diabetes Mellitus. J Diabetes Metab 5: 356 doi:10.4172/2155-6156.1000356

Page 4 of 7

\begin{tabular}{|c|c|c|}
\hline \multirow{2}{*}{ Time(hr) } & Non Diabetic & Diabetic \\
\cline { 2 - 3 } & conc $(\boldsymbol{\mu g} / \mathrm{ml})$ & $0 \pm 0$ \\
\hline 0.5 & 0 & $2.0157 \pm 0.31452$ \\
\hline 1 & $1.03049 \pm 0.34364$ & $1.15479 \pm 0.21450$ \\
\hline 2 & $0.70300 \pm 0.21542$ & $0.81124 \pm 0.34001$ \\
\hline 8 & $0.60084 \pm 0.22686$ & $0.75146 \pm 0.30092$ \\
\hline 24 & $0.43687 \pm 0.22446$ & $0.64006 \pm 0.25617$ \\
\hline
\end{tabular}

Mean \pm SD

Table 1: Mean serum concentration of pioglitazone in non diabetic and diabetic rabbits.
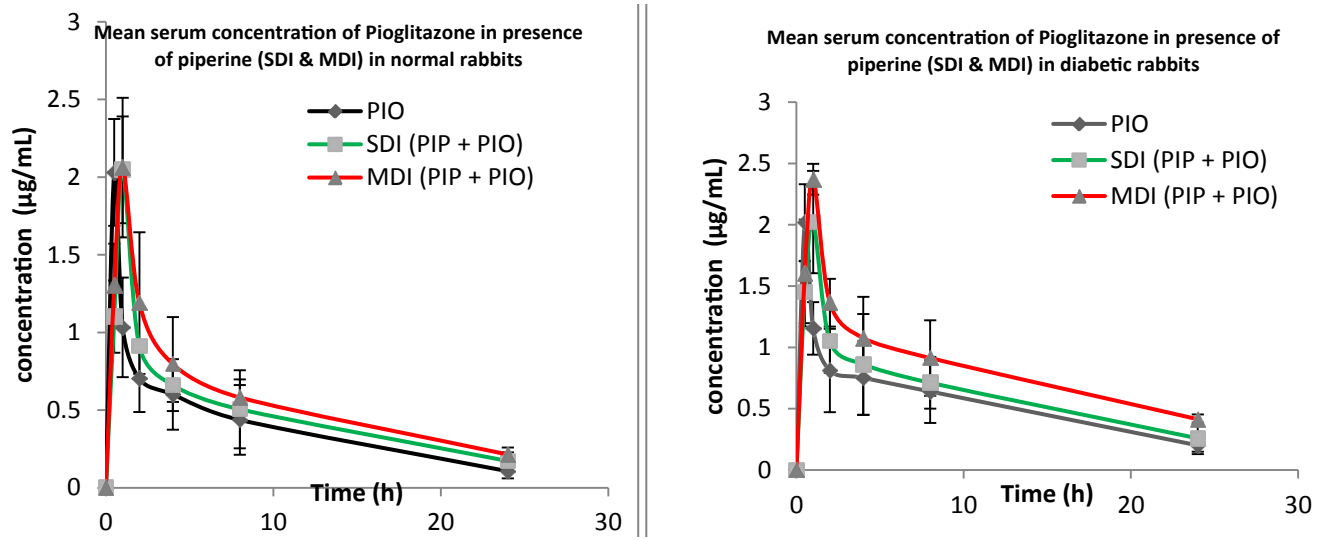

SDI: Single Dose Interaction; MDI: Multiple dose interaction; PIO: pioglitazone; PIP: Piperine

Figure 2: Mean serum concentration of pioglitazone in presence of piperine (SDI\&MDI) in non diabetic and diabetic rabbits.

\begin{tabular}{|c|c|c|c|c|c|c|}
\hline \multirow[b]{2}{*}{ Time(hr) } & \multicolumn{3}{|c|}{ Non Diabetic groups } & \multicolumn{3}{|c|}{ Diabetic groups } \\
\hline & PIO conc $(\mu \mathrm{g} / \mathrm{ml})$ & $\begin{array}{l}\text { PIO+PIP(SDI) } \\
\text { conc }(\mu \mathrm{g} / \mathrm{ml})\end{array}$ & $\begin{array}{l}\text { PIO+PIP(MDI) } \\
\text { conc }(\mu \mathrm{g} / \mathrm{ml})\end{array}$ & PIO conc $(\mu \mathrm{g} / \mathrm{ml})$ & PIO+PIP(SDI)conc $(\mu \mathrm{g} / \mathrm{ml})$ & PIO+PIP(MDI)conc $(\mu \mathrm{g} / \mathrm{ml})$ \\
\hline 0 & $0 \pm 0$ & $0 \pm 0$ & $0 \pm 0$ & $0 \pm 0$ & $0 \pm 0$ & $0 \pm 0$ \\
\hline 0.5 & $2.03049 \pm 0.34364$ & $1.10248 \pm 0.23279$ & $1.30059 \pm 0.27096$ & $2.0157 \pm 0.31452$ & $1.45113 \pm 0.25413$ & $1.60577 \pm 0.43609$ \\
\hline 1 & $1.03281 \pm 0.32064$ & $2.04719 \pm 0.34402^{*}$ & $2.06129 \pm 0.44944^{*}$ & $1.15479 \pm 0.21450$ & $2.02210 \pm 0.41733$ & $2.37158 \pm 0.12586^{*}$ \\
\hline 2 & $0.70300 \pm 0.21542$ & $0.91143 \pm 0.03463$ & $1.18931 \pm 0.45600^{*}$ & $0.81124 \pm 0.34001$ & $1.05065 \pm 0.25901$ & $1.36422 \pm 0.19487^{*}$ \\
\hline 4 & $0.60084 \pm 0.22686$ & $0.65990 \pm 0.10656$ & $0.79644 \pm 0.30285$ & $0.75146 \pm 0.30092$ & $0.86054 \pm 0.41098$ & $1.07525 \pm 0.33711^{*}$ \\
\hline 8 & $0.43687 \pm 0.22446$ & $0.50552 \pm 0.25166$ & $0.58139 \pm 0.11657$ & $0.64006 \pm 0.25617$ & $0.71124 \pm 0.21093$ & $0.91304 \pm 0.30786$ \\
\hline 24 & $0.10687 \pm 0.04611$ & $0.17253 \pm 0.05599$ & $0.21439 \pm 0.04516$ & $0.20100 \pm 0.05145$ & $0.25811 \pm 0.12881$ & $0.41383 \pm 0.03985$ \\
\hline
\end{tabular}

Mean \pm SD; ${ }^{* *}$ significant at $p<0.001 ;{ }^{* *}$ significant at $p<0.01$; *significant at $p<0.05$ compared to pioglitazone control; SDI: Single Dose Interaction; MDI: Multiple dose interaction; PIO: pioglitazone; PIP: Piperine.

Table 2: Mean serum concentration of pioglitazone in presence of piperine (SDI\&MDI) in normal rabbits.

pioglitazone. The mean values along with standard deviation were calculated for each time point (Table 1) and a concentration versus time curve was obtained by plotting the mean concentration of pioglitazone on $\mathrm{x}$-axis against the time points on $\mathrm{y}$-axis (Figure 2). The pharmacokinetic parameters for pioglitazone were calculated and showed a Cmax of $2.03049 \pm 0.49 \mu \mathrm{g} / \mathrm{ml}, \mathrm{T}_{\max }$ of $0.5 \mathrm{hrs}$ and $\mathrm{AUC}_{0 \text { ton }}$ of $9.21 \pm 1.12 \mu \mathrm{g} / \mathrm{hr} \mathrm{ml}$ (Table 2 ) in normal rabbits.

Pharmacokinetics of pioglitazone under piperine pretreatment in normal rabbits

The mean serum concentration levels of pioglitazone in normal rabbits under piperine pretreatment were also read out (Table 3), a comparative concentration versus time curves of all groups was obtained (Figure 3) and the pharmacokinetic parameters of all groups were also compared (Table 4). In normal rabbits, pretreatment of piperine resulted in an increase in mean serum concentration of pioglitazone at $1 \mathrm{hr}$ in single dose interaction group (from $1.03 \pm 0.32$ to $2.04 \pm 0.34 ; \mathrm{p}<0.05$ ); at $1 \& 2 \mathrm{hr}$ in multiple dose interaction group (from $1.03 \pm 0.32$ to 2.06 $\pm 0.44 \&$ from $0.70 \pm 0.21$ to $1.18 \pm 0.45$ respectively; $\mathrm{p}<0.05$ Table 2 ) and significant changes in pharmacokinetic parameters. The $\mathrm{T}_{\max }$ of pioglitazone extended from $0.5 \mathrm{hr}$ to $1 \mathrm{hr}$ under piperine pretreatment.

Pharmacokinetics of pioglitazone in non diabetic and diabetic rabbits with piperine

The pharmacokinetic parameters for pioglitazone were calculated and showed a $\mathrm{C}_{\max }$ of $2.01 \pm 0.87 \mu \mathrm{g} / \mathrm{ml}, \mathrm{T}_{\max }$ of $0.5 \mathrm{hrs}$ and $\mathrm{AUC}_{\text {oton }}$ of $12.65 \pm 1.97 \mu \mathrm{g} / \mathrm{hr} \mathrm{ml}$ in normal rabbits. The $\mathrm{T}_{\max }$ of pioglitazone extended from $0.5 \mathrm{hr}$ to $1 \mathrm{hr}$ under piperine pretreatment. In diabetic rabbits, multiple dose pretreatment of piperine resulted in an increase in mean serum concentration levels of pioglitazone at $2 \mathrm{hr}$ (from $0.81 \pm$ 0.34 to $1.36 \pm 0.19 ; \mathrm{p}<0.05$ Table 3 ) and a significant increase in $\mathrm{AUC}_{0 \text { to }}$ n (from $12.65 \pm 2.97$ to $19.70 \pm 3.16 ; \mathrm{p}<0.05$ Table 3 ). Significant changes 
Citation: Neerati P, Yakkanti AS (2014) Influence of Piperine on Pioglitazone Metabolism and Pk/Pd: Diabetes Mellitus. J Diabetes Metab 5: 356 doi:10.4172/2155-6156.1000356

Page 5 of 7

\begin{tabular}{|c|c|c|c|c|c|c|}
\hline \multirow{2}{*}{ Pk parameter } & \multicolumn{3}{|c|}{ Non Diabetic groups } & \multicolumn{3}{|c|}{ Diabetic Groups } \\
\hline & Pioglitazone & PIO+PIP(SDI) & PIO+PIP(MDI) & Pioglitazone & PIO+PIP(SDI) & PIO+PIP(MDI) \\
\hline $\mathrm{Cmax}(\mu \mathrm{g} / \mathrm{mL})$ & $2.03 \pm 0.49$ & $2.04 \pm 0.17$ & $2.06 \pm 0.28$ & $2.01 \pm 0.87$ & $2.02 \pm 0.17$ & $2.37 \pm 0.31$ \\
\hline Tmax (hrs) & $0.5 \pm 0$ & $1 \pm 0$ & $1 \pm 0$ & $0.5 \pm 0$ & $1 \pm 0$ & $1 \pm 0$ \\
\hline AUC0 to $\mathrm{n}(\mu \mathrm{g} / \mathrm{hr} \mathrm{mL})$ & $9.21 \pm 1.12$ & $11.29 \pm 4.97^{*}$ & $13.32 \pm 2.14$ * & $12.65 \pm 1.97$ & $14.90 \pm 2.11$ & $19.70 \pm 3.16^{*}$ \\
\hline AUC total $(\mu \mathrm{g} / \mathrm{hr} \mathrm{mL})$ & $10.44 \pm 3.07$ & $13.86 \pm 2.07$ & $16.62 \pm 5.13$ * & $15.54 \pm 3.12$ & $19.03 \pm 2.28$ * & $28.31 \pm 3.17$ ** \\
\hline AUMC0 to $n\left(\mu \mathrm{g} / \mathrm{hr} r^{*} \mathrm{hr} \mathrm{mL}\right)$ & $71.22 \pm 15.10$ & $93.41 \pm 11.71$ & $111.48 \pm 14.18^{\star \star}$ & $111.30 \pm 17.37$ & $132.20 \pm 12.73^{*}$ & $185.38 \pm 16.11^{* *}$ \\
\hline AUMC total $\left(\mu \mathrm{g} / \mathrm{hr} r^{\star} \mathrm{hr} \mathrm{mL}\right)$ & $115.09 \pm 26.94$ & $193.44 \pm 21.18$ & $241.45 \pm 28.01^{* *}$ & $222.53 \pm 23.16$ & $297.57 \pm 27.49^{*}$ & $570.43 \pm 32.04^{* *}$ \\
\hline $\mathrm{T} 1 / 2$ (hrs) & $7.98 \pm 1.14$ & $10.32 \pm 0.91^{*}$ & $10.71 \pm 2.13$ * & $9.98 \pm 2.17$ & $11.09 \pm 1.17$ & $14.37 \pm 1.54^{* *}$ \\
\hline MRT (hrs) & $11.01 \pm 2.17$ & $13.94 \pm 1.98$ & $14.52 \pm 2.54$ * & $14.31 \pm 1.17$ & $15.62 \pm 0.16$ & $20.14 \pm 0.91$ * \\
\hline $\mathrm{Cl}(\mathrm{ml} / \mathrm{hr})$ & $0.95 \pm 0.11$ & $0.72 \pm 0.17$ & $0.60 \pm 0.14^{*}$ & $0.64 \pm 0.15$ & $0.52 \pm 0.13$ & $0.35 \pm 0.02$ * \\
\hline $\mathrm{Vd}(\mathrm{ml})$ & $11.02 \pm 3.96$ & $10.74 \pm 2.15$ & $9.29 \pm 1.57$ & $9.26 \pm 1.96$ & $8.41 \pm 1.05$ & $7.32 \pm 0.97$ \\
\hline Vdss (mL) & $10.54 \pm 3.93$ & $10.05 \pm 2.93$ & $8.73 \pm 2.47$ & $9.20 \pm 2.01$ & $8.20 \pm 1.28$ & $7.11 \pm 1.14$ \\
\hline
\end{tabular}

Mean $\pm S D ;{ }^{* * *}$ significant at $p<0.001 ;{ }^{* *}$ significant at $p<0.01$; *significant at $p<0.05$ compared to pioglitazone control; SDI: Single Dose Interaction; MDI: Multiple Dose Interaction; PIO: Pioglitazone; PIP: Piperine.

Table 3: Mean Pharmacokinetic parameters of pioglitazone in presence of piperine (SDI \& MDI) in non diabetic and diabetic rabbits.
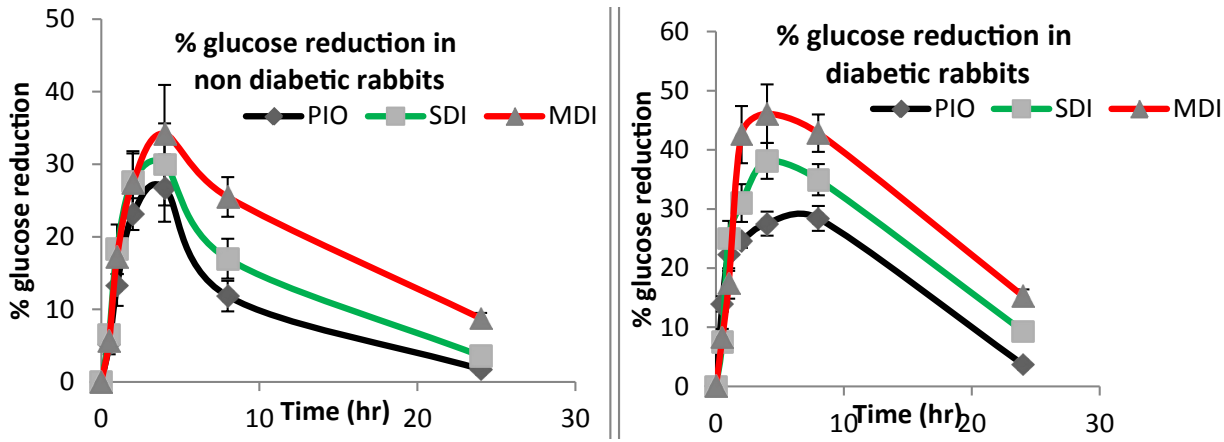

SDI: Single Dose Interaction; MDI: Multiple dose interaction; PIO: pioglitazone; PIP: Piperine.

Figure 3: Comaparative \% glucose reduction in non diabetic and diabetic rabbits.

\begin{tabular}{|c|c|c|c|c|c|c|c|c|c|c|c|c|}
\hline \multirow{3}{*}{$\begin{array}{c}\text { Time } \\
\text { (hr) }\end{array}$} & \multicolumn{6}{|c|}{ NON DIABETIC GROUPS } & \multicolumn{6}{|c|}{ DIABETIC GROUPS } \\
\hline & \multicolumn{2}{|c|}{ PIO } & \multicolumn{2}{|c|}{ PIO+PIP (SDI) } & \multicolumn{2}{|c|}{ PIO+PIP (MDI) } & \multicolumn{2}{|c|}{ PIO } & \multicolumn{2}{|c|}{ PIO+PIP (SDI) } & \multicolumn{2}{|c|}{ PIO+PIP (MDI) } \\
\hline & $\begin{array}{l}\text { Mean glucose } \\
\text { level (mg/dl) }\end{array}$ & $\begin{array}{l}\% \text { glucose } \\
\text { reduction }\end{array}$ & $\begin{array}{l}\text { Mean glucose } \\
\text { level (mg/dl) }\end{array}$ & $\begin{array}{l}\text { Mean glucose } \\
\text { level (mg/dl) }\end{array}$ & $\begin{array}{l}\% \text { glucose } \\
\text { reduction }\end{array}$ & $\begin{array}{l}\text { Mean glucose } \\
\text { level (mg/dl) }\end{array}$ & $\begin{array}{l}\% \text { glucose } \\
\text { reduction }\end{array}$ & $\begin{array}{l}\text { Mean glucose } \\
\text { level (mg/dl) }\end{array}$ & $\begin{array}{l}\% \text { glucose } \\
\text { reduction }\end{array}$ & $\begin{array}{l}\% \text { glucose } \\
\text { reduction }\end{array}$ & $\begin{array}{l}\text { Mean glucose } \\
\text { level (mg/dl) }\end{array}$ & $\begin{array}{l}\% \text { glucose } \\
\text { reduction }\end{array}$ \\
\hline 0 & $106.75 \pm 11.04$ & $0 \pm 0$ & $116.72 \pm 5.98$ & $361.50 \pm 29.03$ & $0 \pm 0$ & $337.27 \pm 16.42$ & $0 \pm 0$ & $352.55 \pm 12.45$ & $0 \pm 0$ & $0 \pm 0$ & $108.24 \pm 5.17$ & $0 \pm 0$ \\
\hline 0.5 & $101.22 \pm 4.18$ & $5.18 \pm 1.36$ & $109.08 \pm 10.10$ & $311.08 \pm 16.15$ & $13.94 \pm 1.28$ & $312.00 \pm 21.19$ & $7.49 \pm 1.05$ & $323.46 \pm 21.08$ & $8.25 \pm 1.48$ & $6.54 \pm 1.29$ & $102.11 \pm 3.01$ & $5.66 \pm 1.19$ \\
\hline 1 & $92.56 \pm 2.64$ & $13.29 \pm 2.80$ & $95.37 \pm 12.00$ & $281.01 \pm 8.11$ & $22.26 \pm 2.72$ & $252.83 \pm 14.5$ & $25.03 \pm 2.94$ & $291.19 \pm 10.12$ & $17.40 \pm 2.57$ & $18.29 \pm 3.41$ & $89.55 \pm 7.80$ & $17.26 \pm 2.42$ \\
\hline 2 & $82.06 \pm 3.74$ & $23.12 \pm 2.19$ & $84.42 \pm 7.42$ & $272.57 \pm 13.08$ & $24.60 \pm 1.18$ & $232.71 \pm 12.10$ & $31.00 \pm 3.20$ & $202.48 \pm 6.08^{*}$ & $42.56 \pm 4.83^{* *}$ & $27.67 \pm 4.13$ & $78.52 \pm 12.19$ & $27.45 \pm 4.02$ \\
\hline 4 & $78.25 \pm 6.97$ & $26.69 \pm 4.62$ & $81.74 \pm 2.14$ & $262.05 \pm 8.90$ & $27.51 \pm 2.03$ & $208.66 \pm 11.39$ & $38.13 \pm 3.04^{*}$ & $190.00 \pm 8.13^{*}$ & $46.10 \pm 4.95^{\star *}$ & $29.96 \pm 5.65$ & $71.22 \pm 2.51$ & $34.20 \pm 6.73$ \\
\hline 8 & $94.12 \pm 8.91$ & $11.83 \pm 2.11$ & $96.90 \pm 4.90$ & $258.78 \pm 6.97$ & $28.41 \pm 2.11$ & $219.39 \pm 7.05$ & $34.95 \pm 2.65^{\star}$ & $201.57 \pm 2.14$ & $42.82 \pm 3.17^{\star *}$ & $16.98 \pm 2.74$ & $80.65 \pm 3.69$ & $25.48 \pm 2.74^{*}$ \\
\hline 24 & $104.89 \pm 4.52$ & $1.74 \pm 0.25$ & $112.53 \pm 3.10$ & $348.15 \pm 19.18$ & $3.69 \pm 0.13$ & $305.80 \pm 14.3$ & $9.33 \pm 1.04^{*}$ & $298.63 \pm 18.72$ & $15.29 \pm 1.12^{* *}$ & $3.58 \pm 0.39$ & $98.75 \pm 4.09$ & $8.76 \pm 0.74^{*}$ \\
\hline
\end{tabular}

Mean \pm SD; ${ }^{* * *}$ significant at $p<0.001 ;{ }^{* *}$ significant at $p<0.01$; ${ }^{*}$ significant at $p<0.05$ compared to pioglitazone control; SDI: Single Dose Interaction; MDI: Multiple Dose Interaction; PIO: Pioglitazone; PIP: Piperine

Table 4: Mean blood glucose changes in non diabetic and diabetic rabbits after oral administration of pioglitazone, piperine, and their combinations (SDI\&MDI).

were observed in pharmacokinetic parameters including decrease in clearance and increase in $\mathrm{t}_{1 / 2}$ under multiple dose exposure. There was an increase in mean serum levels of pioglitazone in both normal and diabetic rabbits and also on the pharmacokinetic parameters under piperine pretreatment.

\section{Pharmacodynamic data}

Effects of piperine on the pharmacodynamics of pioglitazone in non diabetic and diabetic rabbits: The mean blood glucose levels for each time point in normal rabbits were calculated using glucose oxidase peroxidise method and the percentage glucose reduction at each time point compared to the mean glucose levels at $0 \mathrm{hr}$ was calculated. The mean glucose levels and percentage glucose reduction was compared in piperine pretreatment against control group in normal rabbits. Pretreatment of piperine resulted in an increase in percentage glucose reduction more in $8 \& 24 \mathrm{hr}$ (from 11.83 to $25.48 \& 1.74$ to 8.76 respectively; $\mathrm{p}<0.05$ Table 4 ) in multiple dose group of normal rabbits. The mean glucose levels and percentage glucose reduction was compared in piperine pretreatment against control group in diabetic rabbits. Pretreatment of piperine lead to a significant increase in the percentage glucose reduction at 2, 4, $8 \& 24 \mathrm{hr}$ (from 24.60, 27.51, 28.41 \& 3.69 to $42.56,46.10,42.82 \& 15.29$ respectively; $p<0.001$ Table 4 ) in multiple dose groups.

\section{Discussion}

Diabetes is a chronic metabolic disorder and needs prolonged 
treatment for maintenance of normal blood glucose levels. Diabetes poses several complications like retinopathy, nephropathy, peripheral neuropathy and an underlying high oxidant stress. There is a wide use of herbal medications in diabetes condition, not only for controlling or slowing the progress of the disease, but also for most of its complications as they had antioxidant potential. In the context of diabetes and a high prevalence of herbal usage in diabetes; any of the herbal constituents having an influence on the disposition of antidiabetic agents may influence the control of blood glucose levels. Herb drug interactions are usually under-reported as the practitioner is unaware of concomitant use of herbs. Herbs not only in the form of alternative medicinal preparations, but also as dietary constituents can influence the safety and efficacy of a drug. Piperine is a constituent of black pepper and black pepper is widely used as spice. There is an increasing incidence of diabetes, and pioglitazone is a widely prescribed antidiabetic agent either alone or in combination showing better safety profile among thiazolidinedioes with significantly lower risk of cardiovascular damage [12]. Pioglitazone is known to be metabolised by CYP 2C8, 3A4 enzymes [13] and even the active metabolites of pioglitazone $\mathrm{M}$ III \& M IV (keto \& enol derivatives of pioglitazone) were found to be metabolised by CYP 2C8, 3A4 enzymes and hence, found to have improved bioavailability in presence of CYP inhibitors in both, rats [14] and in human liver microsomes in-vitro [15]. A biochemical evidence has long been reported for piperine as a potent inhibitor of drug metabolism [16]. In a study performed in rats, piperine was found to inhibit p-gp and CYP 3A4 activity. The influence of piperine on human hepatic metabolism can be further appreciated in a study on human volunteers, where piperine pretreatment significantly increased the bioavailability of metronidazole, a substrate of CYP 2C8, 2C9 \& 3A4 enzymes [17]. Thus the present study was planned to investigate the influence of piperine on the pharmacokinetics and pharmacodynamics of pioglitazone in rabbits in-vivo, in both normal and alloxan induced diabetic condition. We studied the influence of piperine on the pharmacokinetic \& pharmacodynamics of pioglitazone in rabbits. The pharmacokinetic parameters were found to alter in curcumin pre-treatment, but were statistically more significant in multiple doses pre-treatment. In normal rabbits, pre-treatment of piperine resulted in an increase in $\mathrm{AUC}_{\text {tot }}$ by 0.59 folds $(\mathrm{p}<0.05)$, AUMC $C_{\text {tot }}$ by 1.09 folds $(\mathrm{p}<0.01), \mathrm{t}_{1 / 2}$ by 0.34 folds $(\mathrm{p}<0.05)$, MRT by 0.31 folds $(\mathrm{p}<0.05)$ and a decrease in $\mathrm{Cl}$ by 0.36 folds $(\mathrm{p}<0.05)$. In diabetic rabbits, multiple dose pretreatment of piperine resulted in an increase in $\mathrm{AUC}_{\text {tot }}$ by 0.82 folds $(\mathrm{p}<0.01), \mathrm{AUMC}_{\text {tot }}$ by 1.56 folds $(\mathrm{p}<0.01), \mathrm{t}_{1 / 2}$ by 0.43 folds $(\mathrm{p}<0.01)$, MRT by 0.40 folds $(\mathrm{p}<0.05)$ and a decrease in $\mathrm{Cl}$ by 0.45 folds $(\mathrm{p}<0.05)$. Maximum percentage glucose reduction was observed at $4 \mathrm{hr}$ in all groups of normal rabbits and any changes were less significant. In diabetic rabbits also maximum percentage glucose reduction was observed at $4 \mathrm{hr}$ and the influence of piperine was found to be statistically significant in both single dose pretreatment (at 4,8 , $24 \mathrm{hr}$ by $0.38,0.23,1.52$ folds respectively; $\mathrm{p}<0.05$ ) and multiple dose pretreatment (at 2, 4, 8, $24 \mathrm{hr}$ by $0.73,0.67,0.50,3.14$ folds respectively; $\mathrm{p}<0.01)$. The increase in AUC only in multiple dose treatment suggests an inhibitory influence of piperine on hepatic metabolism. Also, influence of piperine being effective in improving pharmacodynamics (glycemic control) only in diabetic rabbits indicates that the alteration might be partly because of improved pharmacokinetics of pioglitazone and partly because of antihyperglycemic activity of piperine. Thus, the improved pharmacokinetic parameters of pioglitazone was more observed in the multiple dose treatment groups, and the improvement of pharmacodynamics was significant in only diabetic rabbits under multiple dose treatment, thus showing the significance of influence of piperine in multiple dose exposure.

\section{Conclusions}

The results of increased AUC of pioglitazone under piperine exposure suggest an interaction which may be due to decreased metabolism of pioglitazone as a result of CYP 3A4 and 2C8 inhibition. Since the alterations are more pronounced in multiple dose treatment groups, it indicates the significance of long term exposure of piperine in diabetic condition being controlled by pioglitazone in rabbits, and thus it may apply to diabetic patients under pioglitazone treatment. Hence, the combination has a beneficial effect in diabetic condition, but therapeutic drug monitoring has to be observed in view of side effects of pioglitazone. Hence the present investigation warrants further studies to find out the relevance of this interaction in human beings and postulates the exact mechanism involved.

\section{Acknowledgement}

This research work was funded by All India Council for Technical Education (AICTE), New Delhi, India. The authors are thankful AICTE (8023/BOR/RID/RPS 107/2009-10)

\section{References}

1. Bhardwaj RK, Glaeser H, Becquemont L, Klotz U, Gupta SK, et al. (2002) Piperine, a major constituent of black pepper, inhibits human P-glycoprotein and CYP3A4. J Pharmacol Exp Ther 302: 645-650.

2. Atal CK, Dubey RK, Singh J (1985) Biochemical basis of enhanced drug bioavailability by piperine: evidence that piperine is a potent inhibitor of drug metabolism. J Pharmacol Exp Ther 232: 258-262.

3. Reen RK, Jamwal DS, Taneja SC, Koul JL, Dubey RK, et al. (1993) Impairment of UDP-glucose dehydrogenase and glucuronidation activities in liver and small intestine of rat and guinea pig in vitro by piperine. Biochem Pharmacol 46 229-238.

4. Eapen SMS, Grampurohit ND (2002) Chemical Evaluation of Navayasa churna. Indian Drugs 39: 101-105.

5. Atal S1, Agrawal RP, Vyas S, Phadnis P, Rai N (2012) Evaluation of the effect of piperine per se on blood glucose level in alloxan-induced diabetic mice. Acta Pol Pharm 69: 965-969.

6. Scheen AJ (2007) Pharmacokinetic interactions with thiazolidinediones. Clin Pharmacokinet 46: 1-12.

7. King H, Aubert RE, Herman WH (1998) Global burden of diabetes, 1995-2025 prevalence, numerical estimates, and projections. Diabetes Care 21: 1414 1431.

8. Kolte BL1, Raut BB, Deo AA, Bagool MA, Shinde DB (2003) Liquid chromatographic method for the determination of rosiglitazone in human plasma. J Chromatogr B Analyt Technol Biomed Life Sci 788: 37-44.

9. Sudhir N, Umathe, Pankaj V, Dixit Vijendra kumar, Kuldeep U, et al. (2008) Quercetin pretreatment increases the bioavailability of pioglitazone in rats: Involvement of CYP3A inhibition. Biochemical Pharmacology 75: 1670-1676.

10. Shukla R, Anan K, Prabhu KM, Murlly PS (1994) Hypoglycemic effect of the water extract of ficus bengalensis in alloxan recovered, mildy diabetic and severely diabetic rabbits. Int J Diab Dev:78-81.

11. Trinder $P$ (1969) Determination of blood glucose using an oxidase-peroxidase system with a non-carcinogenic chromogen. J Clin Pathol 22: 158-161.

12. Lincoff AM1, Wolski K, Nicholls SJ, Nissen SE (2007) Pioglitazone and risk of cardiovascular events in patients with type 2 diabetes mellitus: a meta-analysis of randomized trials. JAMA 298: 1180-1188.

13. Scheen AJ (2007) Pharmacokinetic interactions with thiazolidinediones. Clin Pharmacokinet 46: 1-12.

14. Deng LJ1, Wang F, Li HD (2005) Effect of gemfibrozil on the pharmacokinetics of pioglitazone. Eur J Clin Pharmacol 61: 831-836. 
Citation: Neerati P, Yakkanti AS (2014) Influence of Piperine on Pioglitazone Metabolism and Pk/Pd: Diabetes Mellitus. J Diabetes Metab 5: 356 doi:10.4172/2155-6156.1000356

Page 7 of 7

15. Jaakkola T1, Laitila J, Neuvonen PJ, Backman JT (2006) Pioglitazone is metabolised by CYP2C8 and CYP3A4 in vitro: potential for interactions with CYP2C8 inhibitors. Basic Clin Pharmacol Toxicol 99: 44-51

16. Atal CK, Dubey RK, Singh J (1985) Biochemical basis of enhanced drug bioavailability by piperine: evidence that piperine is a potent inhibitor of drug metabolism. J Pharmacol Exp Ther 232: 258-262.
17. Amar Singh, Vivek Kumar P, VikashJ, Minoo H, Parabia RA, GauravS ( 2010) In - Vivo Assessment of Enhanced Bioavailability of Metronidazole with Piperine in Rabbits. Research Journal of Pharmaceutical Biological and Chemical Sciences 1: 273-278. 